Kennesaw State University

DigitalCommons@Kennesaw State University

Faculty Publications

11-2009

\title{
Submonthly Polar Vortex Variability and Stratosphere-Troposphere Coupling in the Arctic
}

Robert X. Black

Georgia Institute of Technology - Main Campus

Brent A. McDaniel

Kennesaw State University, bmcdanie@kennesaw.edu

Follow this and additional works at: https://digitalcommons.kennesaw.edu/facpubs

\section{Recommended Citation}

Black RX and McDaniel BA. 2009. Submonthly polar vortex variability and stratosphere-troposphere coupling in the arctic. J Climate 22:5886-901.

This Article is brought to you for free and open access by DigitalCommons@Kennesaw State University. It has been accepted for inclusion in Faculty Publications by an authorized administrator of DigitalCommons@Kennesaw State University. For more information, please contact digitalcommons@kennesaw.edu. 


\title{
Submonthly Polar Vortex Variability and Stratosphere-Troposphere Coupling in the Arctic
}

\author{
ROBERT X. BLACK \\ School of Earth and Atmospheric Sciences, Georgia Institute of Technology, Atlanta, Georgia \\ Brent A. MCDANIEL \\ Department of Biology and Physics, Kennesaw State University, Kennesaw, Georgia
}

(Manuscript received 17 July 2008, in final form 16 March 2009)

\begin{abstract}
A principal component analysis is performed to characterize intraseasonal variability in the boreal stratospheric polar vortex. In contrast to previous studies, the current analysis examines daily zonal-mean variability within a limited spatial domain encompassing the stratospheric polar vortex. The leading EOFs are vertically coherent north-south dipoles in the zonal-mean zonal wind extending through the lower stratosphere. The first mode represents variability in polar vortex strength and is highly correlated with the stratospheric northern annular mode (SNAM). The second mode, the polar annular mode (PAM), represents variability in the latitudinal position of the polar vortex and possesses a poleward-retracted dipole anomaly structure. Composite analyses indicate that large-amplitude PAM events are relatively short lived (1-2 weeks) compared to SNAM events (1 month or longer). Trend analyses further reveal that recent decadal trends in the boreal polar vortex project more strongly onto PAM than SNAM.

Composite analyses illustrate that the time evolution of sudden stratospheric warming events is dominated by SNAM, whereas SNAM and PAM play approximately equal roles in final warming events. Linear regression analyses reveal that SNAM and PAM result in circumpolar circulation and temperature anomalies of similar magnitudes within the high-latitude troposphere. It is concluded that PAM represents a previously unrecognized annular mode that strongly couples the stratosphere and troposphere on submonthly time scales at mid- to high latitudes. It is further suggested that the SNAM/PAM framework provides a means for isolating the proximate tropospheric response to respective variations in the strength and position of the stratospheric polar vortex.
\end{abstract}

\section{Introduction}

It is now well established that robust stratospheretroposphere coupling occurs during boreal winter in connection with intraseasonal variations in the northern annular mode (NAM; see Thompson and Wallace 1998; Baldwin and Dunkerton 2001; McDaniel and Black 2005). This coupling is associated with a vertically coherent zonal wind anomaly pattern extending from the earth's surface upward into the middle stratosphere. At stratospheric altitudes the NAM is manifested by variations in the strength of the stratospheric polar vortex

\footnotetext{
Corresponding author address: Dr. Robert X. Black, School of Earth and Atmospheric Sciences, Georgia Institute of Technology, Atlanta, GA 30332-0340.

E-mail: rob.black@eas.gatech.edu
}

("pulsing"), while at tropospheric altitudes the NAM is interpreted as a north-south "wobble" in the position of the midlatitude jet stream (Wittman et al. 2005). The NAM is linked to important large-scale circulation anomalies in the extratropical troposphere impacting regional weather conditions (Thompson and Wallace 2001).

Although midwinter weakenings of the polar vortex, known as sudden stratospheric warming (SSW) events, are linked to intraseasonal NAM variability (Limpasuvan et al. 2004), this relationship is not one to one (Charlton and Polvani 2007). Furthermore, there are distinctions among SSW events in their respective impact on the stratospheric polar vortex. Charlton and Polvani (2007) find that just under $50 \%$ of SSW events result in a "splitting" of the polar vortex (wavenumber 2), while the remainder lead to polar vortex "displacement" 
(wavenumber 1). Vortex splitting events are typically preceded by a period of stratospheric "preconditioning," during which time the polar vortex strengthens and retracts poleward. Interestingly, the net tropospheric impact of SSW events is nonetheless found to be similar for both the splitting and displacement events (Charlton and Polvani 2007).

In addition to midwinter polar vortex weakenings, each winter season concludes with a rather abrupt transition from circumpolar stratospheric westerly winds to easterlies. This annual breakdown of the polar vortex is known as the stratospheric final warming (SFW). A recent observational study of SFW events found that these events strongly organize the large-scale circulation of the stratosphere and troposphere (Black et al. 2006, hereafter BMR). Specifically, SFW events are associated with a vertically coherent north-south dipole pattern in the zonal wind anomaly field at mid- to high latitudes extending from the middle stratosphere downward into the troposphere. However, this pattern is distinct from the canonical NAM structure because the primary centers in the north-south anomaly dipole are retracted northward compared to the NAM.

SFW events exhibit a robust bidirectional dynamical coupling of the stratosphere and troposphere (Black and McDaniel 2007). The evolution is characterized by an anomalous upward Eliassen-Palm (E-P) signature at high latitudes extending from the surface to the middle stratosphere. This is followed by direct and indirect feedbacks of the stratospheric annular circulation upon the high-latitude troposphere (Black and McDaniel 2007). As for SSW splitting events, SFW events are preceded by a preconditioning of the stratospheric zonal-mean flow. Conversely, the zonally asymmetric (wave) evolution of SFW events (characterized by a strong wavenumber-1 component) more closely resembles SSW displacement events. Thus, SFW events have notable structural and dynamical distinctions from both SSW events and subseasonal NAM variability.

Studies of long-term variability in the Arctic climate have revealed a paradox in which there has been an apparent decoupling between the Arctic Oscillation (AO; the near-surface manifestation of the NAM) and high-latitude climate (Overland and Wang 2005), which were previously considered to be intrinsically linked (e.g., Thompson et al. 2000). This leaves residual highlatitude climate variability that is unaccounted for by the primary extratropical modes of climate variability, such as the AO/NAM and Pacific-North American pattern (e.g., Wu and Karoly 2007). Recent studies of highlatitude tropospheric climate variability reveal the existence of higher-order annular modes [e.g., the "central Arctic" pattern of Maslanik et al. (2007), and Arctic mode rotated principal component 2 (RPC2) of Bromwich and Wang (2008)] that provide important impacts upon Arctic climate. Similar to SFW events, these modes are more circumpolar and northward retracted in comparison to the canonical AO-NAM signatures.

The results discussed above indicate that SFW (and some SSW) events may be linked to distinct and previously unrecognized annular modes of variability at high latitudes, with potential implications for Arctic climate. Here we explore this idea more rigorously by characterizing the primary modes of intraseasonal variability in the wintertime stratospheric polar vortex. In contrast to previous studies (Nigam 1990; Lorenz and Hartmann 2003; Itoh and Harada 2004; Song et al. 2006), the current analysis concentrates on zonal-mean variability on short (daily) time scales within a limited spatial domain (the lower stratosphere at high latitudes) encompassing the stratospheric polar vortex. This analysis leads to the identification of a previously unrecognized intraseasonal annular mode that is orthogonal to the stratospheric NAM and strongly couples the stratosphere and troposphere on submonthly time scales at mid- to high latitudes. Regression analyses reveal that this mode induces circumpolar circulation and temperature anomalies within the high-latitude troposphere. Our paper has the following structure: section 2 outlines the details of our methodological approach; the fundamental structures and vertical connections are presented in section 3 ; the statistical robustness of our fundamental results is tested in section 4 , while variability on intraseasonal and interannual time scales is examined in sections 5 and 6 , respectively; finally, a summary and concluding remarks are provided in section 7 .

\section{Data and methods}

The basic dataset employed consists of $49 \mathrm{yr}$ (1958-2006) of National Centers for Environmental Prediction-National Center for Atmospheric Research (NCEP-NCAR) daily averaged reanalyses (Kalnay et al. 1996) archived on 17 pressure levels extending from 1000 to $10 \mathrm{hPa}$. Daily anomalies in the field variables are taken as deviations from a smoothed climatological annual cycle. Following Black and McDaniel (2007), we construct the annual cycle by temporal sequencing of the long-term mean values for each calendar day. This repeating annual cycle is then smoothed by performing a harmonic analysis and retaining the first six Fourier harmonics. Daily variability in the polar vortex is characterized by performing a principal component (PC) analysis of daily anomalies in zonal wind and geopotential height. We first consider anomalies in the zonal-mean zonal wind field in the lower stratosphere at 
high latitudes. More specifically, we perform the PC analysis over a domain (illustrated graphically in Fig. 1c) encompassing the polar vortex in the lower stratosphere $\left(45^{\circ}-90^{\circ} \mathrm{N}\right.$ and $\left.100-10 \mathrm{hPa}\right)$. The analysis considers the months of January-April (JFMA), during which time the polar vortex is highly variable and most SFW events typically occur (BMR). The input zonal wind anomalies are weighted latitudinally (by square root of the cosine of latitude) and normalized in the vertical [by the longterm and areal-averaged standard deviation at each level, following Baldwin and Dunkerton (1999)]. A principal component analysis is then performed to identify the dominant empirical orthogonal functions (EOFs) and PC time series.

The resulting PC time series for each EOF is normalized for the same time period (January-April) and then used as a basis for performing composite, linear regression, power spectrum, and trend analyses. In pursuing the composite analyses, discrete events are identified using running 5-day means of the respective PC time series. Otherwise, all of the analyses presented and discussed employ unfiltered daily anomalies in the field variables and time series considered. The robustness of the basic $\mathrm{PC}$ results are tested by performing a series of sensitivity analyses that examine the dependence upon (i) the spatial domain specification (with respect to latitude and pressure), (ii) the field variable analyzed (zonal wind versus geopotential height), and (iii) the use of zonalmean versus zonally varying quantities. To facilitate direct comparisons with canonical NAM variability, we also derive a separate daily NAM index for each vertical level (as in Baldwin and Dunkerton 2001). This involves first identifying EOFs from the low-frequency intraseasonal height anomaly field and then projecting the daily height anomalies upon the leading EOF (NAM).

The statistical significance of our results is assessed in multiple ways. The statistical significance of correlation and linear regression analyses is tested using a two-sided Student's $t$ test (e.g., Robinson et al. 2006). In this case it is important to account for any serial correlations that may exist in the daily data. To do this we follow the methods of Wilks [2006, their Eq. (5.12)]. As an example, the 50-hPa NAM index exhibits a lag-1 autocorrelation of $\sim 0.98$. Over 49 JFMA periods, this translates into an effective sample size of $\sim 60$ for the purposes of statistical calculations. Similar results are found for other stratospheric levels considered in our study. Because the effective sample size is larger at tropospheric levels (given the weaker memory in the large-scale tropospheric circulation), 60 represents an effective lower bound on the effective sample size for many of our analyses. The statistical robustness of the individual EOFs identified in our principal component analysis is tested using the eigenvalue

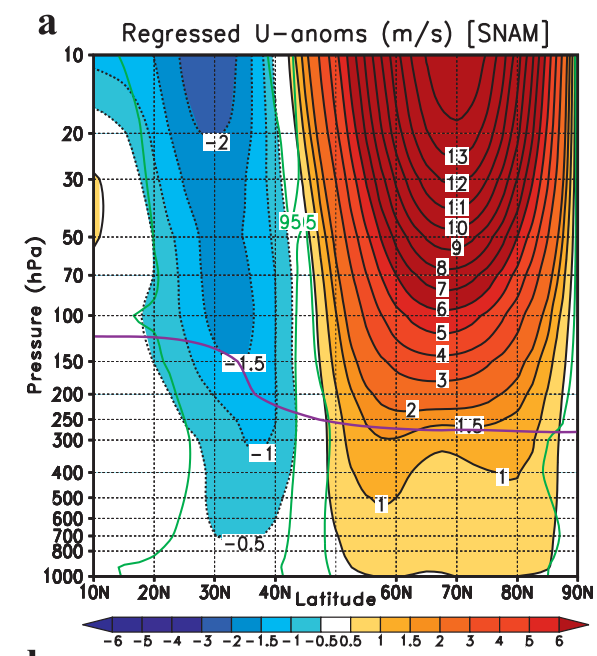

b
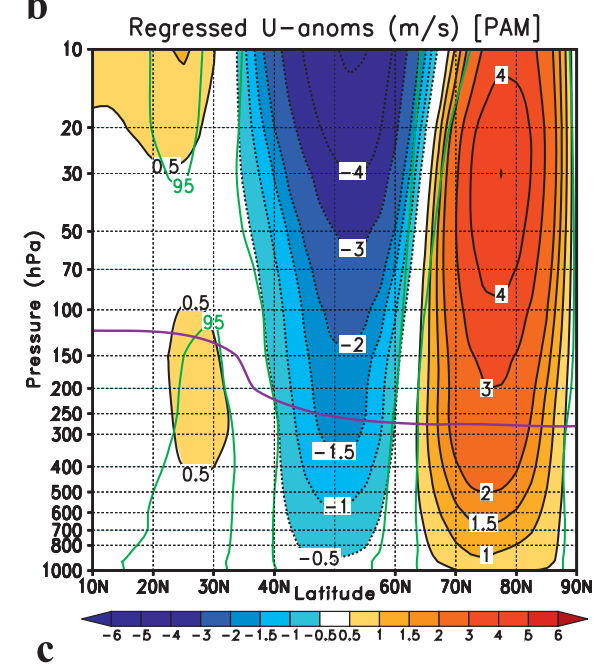

c

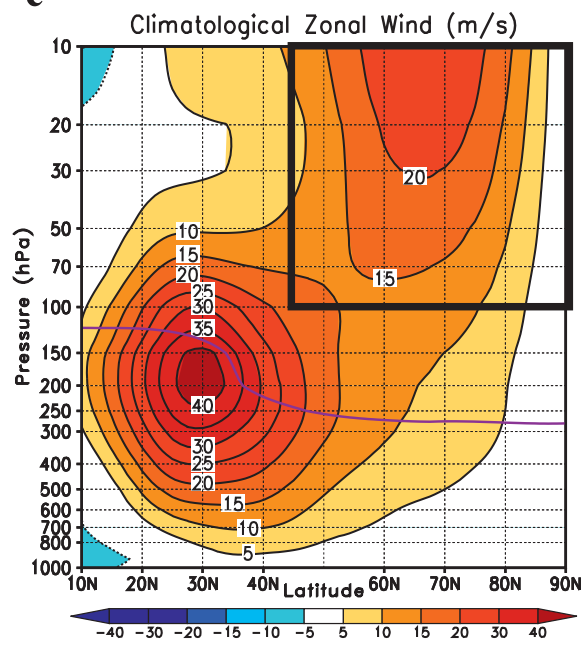

FIG. 1. Zonally averaged zonal wind field $\left(\mathrm{m} \mathrm{s}^{-1}\right)$. Regressions of boreal winter (JFMA) wind anomalies against the daily (a) SNAM and (b) PAM indices. The 95\% confidence level is also plotted in green. (c) For reference, the climatological winter mean is shown, with the region used in the EOF calculation bounded in black. 
separation procedure of North et al. (1982; also see Wilks 2006) using an effective sample size of 60 . The composite index analyses presented in section 5 are tested using a two-sided Student's $t$ test. In this case, each event is treated as an independent sample (since they are required to be well separated in time). Significant values are indicated by thickened color contours in the respective composite time series plots.

\section{Fundamental structures and tropospheric connections}

The leading eigenvectors of the EOF analysis of daily zonal-mean zonal wind anomalies account for $71 \%$ and $15 \%$ of the respective variance in the high-latitude stratosphere. EOF2 is well separated from EOF3 (according to North's rule, see section 2), which explains $5 \%$ of the total variance. The representative zonal-mean zonal wind anomaly structures for the first two EOFs are obtained by regressing the daily zonal wind anomaly field against the respective normalized PC time series for JFMA (Fig. 1). Regions of statistically significant regression features are encompassed using a green contour. EOF1 is a prominent north-south anomaly dipole in the stratospheric zonal wind field with a nodal line in the midlatitudes. Both anomaly poles have significant extensions down into the troposphere. The pattern in the high-latitude stratosphere strongly resembles the NAM (especially at upper levels), and primarily acts to modify the polar vortex strength (cf. Figs. 1a,c). Further, the PC time series for EOF1 is highly correlated with the stratospheric NAM (e.g., a correlation of 0.97 with 50-hPa NAM). However, the tropospheric zonal wind anomaly pattern corresponds less well with the canonical NAM structure (e.g., Fig. 7d of Thompson and Wallace 2000). We attribute these structural differences to the use of unfiltered daily anomalies in our analysis (versus using low-pass data). This permits the isolation of the "instantaneous" tropospheric response before the troposphere has a chance to dynamically adjust (e.g., via the effects of synoptic eddies). Conversely, low-pass data will implicitly include tropospheric structures linked to subsequent dynamical adjustment. Given the strong correspondence with the canonical stratospheric NAM, here we refer to EOF1 as the stratospheric NAM pattern (or SNAM).

The structure of EOF2 exhibits strong parallels with EOF1 except that the north-south anomaly dipole pattern is retracted substantially northward. The northern pole achieves maximum amplitude between $70^{\circ}$ and $80^{\circ} \mathrm{N}$, whereas the southern pole is concentrated in the midlatitudes. Interestingly, these latitude bands overlap the key latitude bands in which BMR observed strong zonal wind changes during SFW events. Because of this high-latitude annular structure, herein we refer to EOF2 as the polar annular mode (PAM). We note prominent and statistically significant downward extensions of the dipole pattern into tropospheric altitudes, especially for the northern pole. In fact, it is interesting to note that the zonal wind signature in the high-latitude troposphere is actually larger in amplitude than its SNAM counterpart (cf. Figs. 1a,b), in stark contrast to the respective stratospheric signatures. The nodal line for PAM is approximately aligned along the axis of the polar vortex. Thus, in contrast to SNAM, PAM represents north-south excursions in the polar vortex position as might occur in association with stratospheric "preconditioning" (Andrews et al. 1987). This behavior is illustrated in Fig. 2, which shows the typical net impact of the two opposing phases of PAM upon the total zonal wind field. We note there is a $10^{\circ}$ latitudinal shift in the polar vortex position between the positive and negative phases of PAM. An analysis of the respective time series reveals, as anticipated, that PAM is effectively uncorrelated with the canonical stratospheric NAM (correlation of less than 0.05). Consequently, we conclude that PAM is a newly identified annular mode that (i) extends over stratospheric and tropospheric altitudes, (ii) is distinct from the stratospheric NAM, and (iii) exhibits a robust circumpolar circulation with a downward extension into the high-latitude troposphere.

The robustness of the dipole patterns depicted in Fig. 1 is tested by performing a correlation analysis between the two primary latitudinal centers of action (poles) for each EOF structure. This is pursued separately for SNAM and PAM along the $50-\mathrm{hPa}$ pressure surface (a key intermediate vertical level that is emphasized throughout our manuscript). To do this, in each case we simply correlate the daily time series of zonal-mean zonal wind at the northern pole with the respective time series at the southern pole for JFMA. For SNAM the basic input is the unfiltered daily zonal-mean zonal wind anomaly field at $30^{\circ}$ and $70^{\circ} \mathrm{N}$, respectively, resulting in a statistically significant correlation of -0.52 . Recognizing the dominance of SNAM in the spectrum of the daily zonal wind variability $(\sim 70 \%)$ over the domain of interest, before performing the parallel correlation analysis for PAM we first explicitly remove that portion of the daily zonal wind variability that is linearly related to SNAM. The residual zonal wind field is then used as input for the time series correlation between $52.5^{\circ}$ and $77.5^{\circ} \mathrm{N}$ (the two poles for PAM). This leads to a statistically significant correlation of -0.67 , indicating that the PAM centers are negatively correlated with one another at a level that is comparable to SNAM. This provides further support to the idea that PAM represents a higher-order dipole structure linked to north-south 


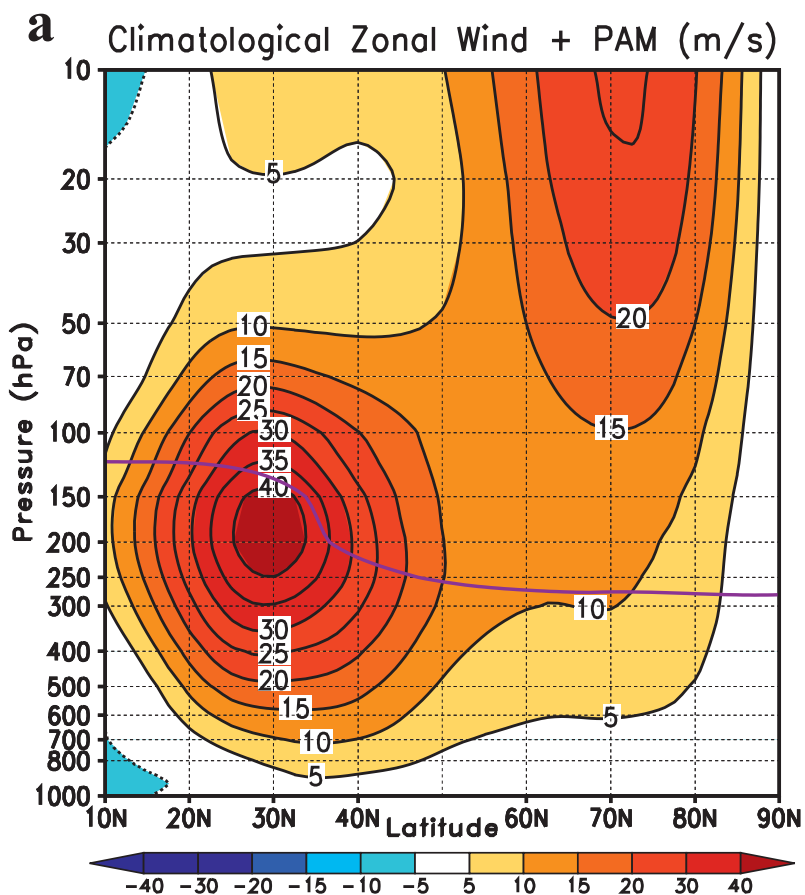

b

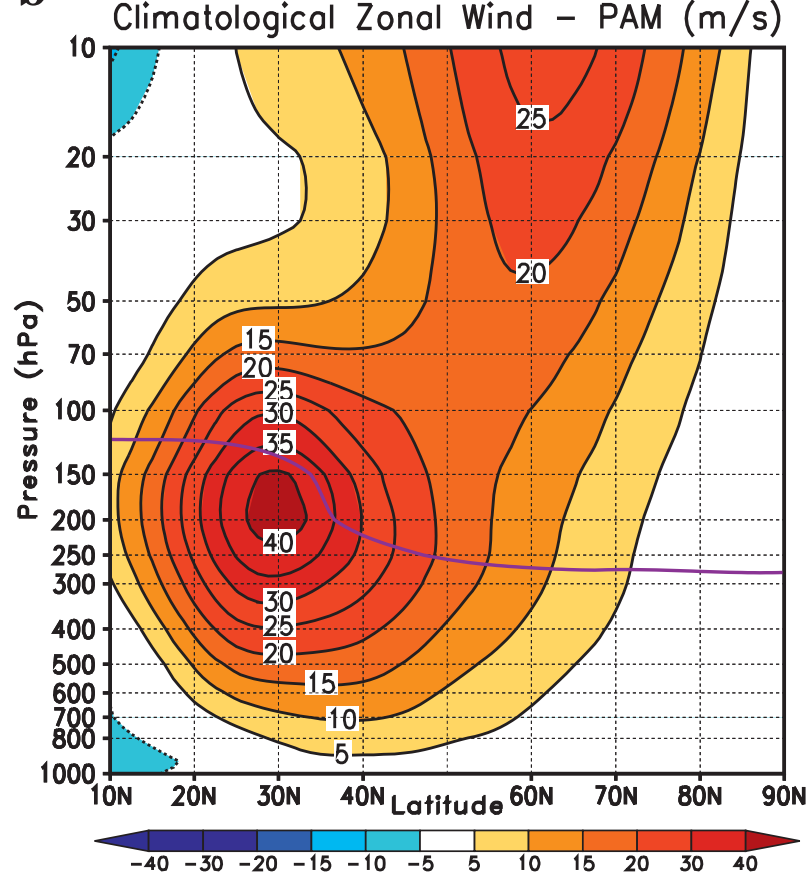

FIG. 2. Zonally averaged zonal wind field $\left(\mathrm{m} \mathrm{s}^{-1}\right)$ illustrating the effect of PAM on the climatological winter mean. The winter mean (a) plus and (b) minus the $1 \sigma$ PAM field.

variations in the latitudinal position of the stratospheric polar vortex.

As discussed above we ascribe the respective behavior of the SNAM and PAM patterns as representing variability in the strength (pulsing) and position (wobbling) of the stratospheric polar vortex. We noted earlier that the interpretation of the canonical NAM's role in the stratosphere is different from its tropospheric impact, where it is considered to modulate the position of the midlatitude (eddy driven) tropospheric jet. Thus, the respective roles of SNAM and PAM in polar vortex variability represent a reordering of the canonical paradigm for the impact of leading annular modes upon the extratropical troposphere (Lorenz and Hartmann 2003; Wittman et al. 2005). This reordering may be related to respective differences in the impact of large-scale Rossby waves on the extratropical tropospheric and stratospheric jet streams (i.e., eddy driven versus "eddy weakened").

The fact that the canonical tropospheric NAM pattern is distinct from the SNAM pattern exhibited in Fig. 1a is consistent with the idea that, during periods of stratosphere-troposphere NAM coupling, the tropospheric NAM represents, at least in part, an internal tropospheric response to stratospheric NAM variability. As such, the tropospheric portion of the EOF1 pattern likely more closely represents the direct impact of the stratospheric NAM upon the troposphere (e.g., McDaniel and Black 2005).

The statistical relationship between the stratospheric and tropospheric NAM is further explored in Fig. 3, which displays vertical profiles of the daily temporal correlation between different stratospheric-based NAM measures (including SNAM) and the canonical NAM time series for each vertical level. For example, the red line is the correlation between the $10-\mathrm{hPa}$ canonical NAM time series and the canonical NAM time series at all other vertical levels (resulting in a value of 1.0 at $10 \mathrm{hPa}$, with a monotonic decrease below). Other stratospheric NAM measures that are considered are the 50-hPa canonical NAM time series (blue), a vertical average of the canonical NAM time series (gold) over the lower stratosphere (from 10 to $100 \mathrm{hPa}$ ), and the SNAM time series (purple). Statistical significance testing (described in section 2) reveals that (i) all of the correlation profiles are significant at stratospheric levels and (ii) correlation magnitudes greater than 0.3 are significant at tropospheric levels.

We first note that the respective vertical profiles for SNAM, the vertically averaged NAM, and the $50-\mathrm{hPa}$ time series are very close to one another (with the latter two being virtually identical), achieving peak correlations near $50 \mathrm{hPa}$. Except for the 10-hPa curve, all of the correlation profiles exceed 0.85 above $150 \mathrm{hPa}$, suggesting a strong linkage between each time series and the canonical NAM in the lower stratosphere. All of the correlation profiles then drop precipitously (by 0.4 or more) between 100 and $300 \mathrm{hPa}$, which encompasses the tropopause layer. Below $300 \mathrm{hPa}$ all of the correlation 


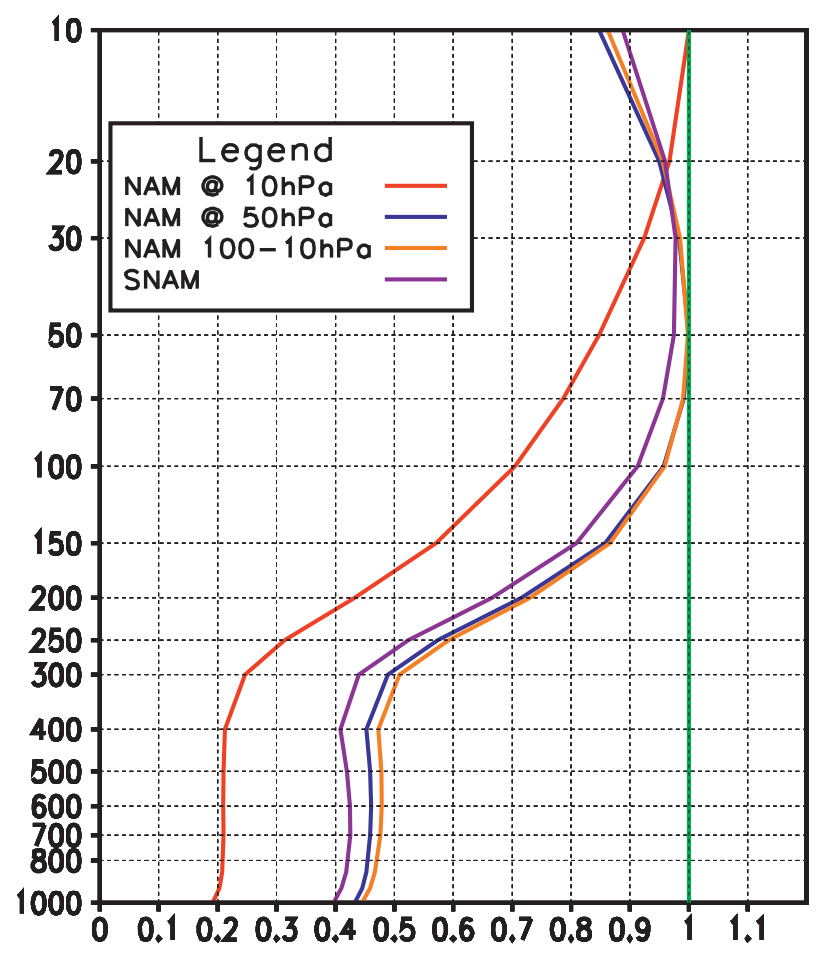

FIG. 3. Multilevel correlations for daily wintertime (JFMA) NAM indices. Each plot represents the correlation of a given index with the canonical NAM index at various levels. See text for details on how the indices were calculated. For reference, the green contour represents perfect correlation.

profiles are less than 0.5 but are relatively uniform. Within the troposphere the $10-\mathrm{hPa}$ profile nears 0.2 (statistically insignificant), while the other three curves fall between 0.4 and 0.5. Considering the 10-hPa NAM time series as representing midstratospheric NAM behavior, our correlation results indicate the following behavior for day-to-day variability in the NAM:

1) strong vertical coherence in the lower stratosphere,

2) substantial decoupling across the tropopause,

3) vertically uniform impact of SNAM upon tropospheric NAM, and

4) poor correlation of midstratospheric NAM with tropospheric NAM;

5) SNAM accounts for less than $25 \%$ of the daily tropospheric NAM variability.

A key result is that daily stratospheric and tropospheric NAM variability is largely decoupled, consistent with the above idea that the proximate tropospheric manifestation of SNAM (as revealed in Fig. 1a) differs from the canonical tropospheric NAM pattern. We have also explored (i) possible lag relationships among the respective NAM time series and (ii) correlations between PAM and the various NAM indices. However, in both cases a null result is obtained because 1) the NAM lag correlation analyses reveal no enhanced relationships and 2) the PAM index exhibits no significant simultaneous or lag correlation with any of the NAM indices considered. Thus, PAM does not appear to have a systematic relationship with either the tropospheric or the stratospheric NAM. The relationship between PAM and NAM during stratospheric warming events will be discussed in section 5 .

We next consider the impact of PAM upon the zonalmean tropospheric circulation. In addition to directly impacting the latitudinal position of the stratospheric polar vortex, Fig. 2 reveals that the opposing phases of PAM provide substantial alterations to the zonal wind structure in the vicinity of the tropopause at mid- to high latitudes. In particular, the positive (negative) PAM phase is characterized by a relatively weak (strong) meridional gradient in zonal wind along the tropopause north of $50^{\circ} \mathrm{N}$. This difference is quite dramatic and is very likely to impact both the forcing and the propagation of tropospheric Rossby wave activity (via respective changes in the instability and refractive index characteristics).

We next isolate the regional (zonally varying) manifestations of SNAM and PAM by performing linear regression analyses of the SNAM and PAM indices against the 50- and 500-hPa geopotential height and near-surface temperature anomaly fields (Fig. 4). Similar to Fig. 1, regions of statistically significant regression features are outlined by a green contour. The analysis of the 50-hPa height field reveals largely zonally symmetric anomaly structures for both SNAM and PAM. For each $\mathrm{EOF}$, the positive phase is associated with circumpolar negative height anomalies that are surrounded by positive height anomalies at lower latitudes. The primary main structural difference is that the PAM anomaly pattern is retracted poleward relative to the SNAM pattern, as would be expected from a consideration of Fig. 1 (in association with geostrophic balance). Also, the SNAM anomaly magnitudes are much larger than those for PAM. The parallel analysis for 500-hPa geopotential height shows qualitatively similar patterns with (i) less zonal symmetry than that at $50 \mathrm{hPa}$ and (ii) comparable magnitudes. Again, the latter feature is consistent with the results presented in Fig. 1. A comparison of the tropospheric expression of SNAM with the canonical tropospheric NAM (e.g., Fig. 1 of Baldwin and Dunkerton 1999) reveals a substantial difference in the relative strength of the North Atlantic anomaly centers, which are much less prominent (versus the polar center) in the current analysis. Although the respective Arctic anomaly centers have similar magnitudes in the two analyses, the local height anomaly minimum over southern Greenland is $\sim 65 \%$ larger in magnitude in the 
Regressed 50hPa Z Anoms (m) (SNAM)

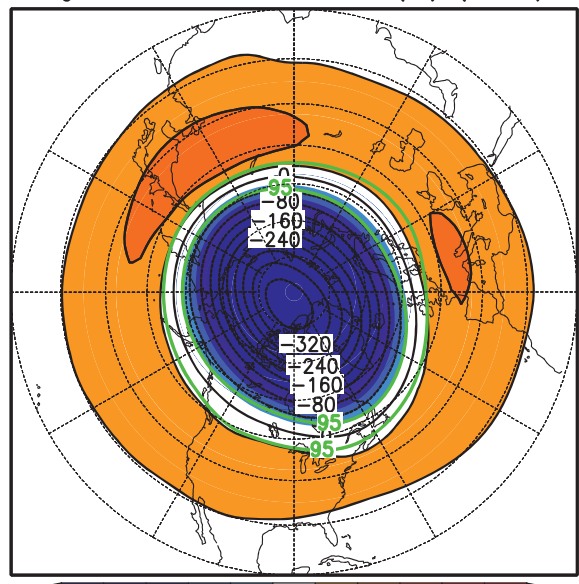

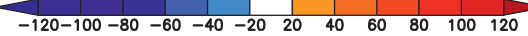

Regressed 500hPa Z Anoms (m) (SNAM)

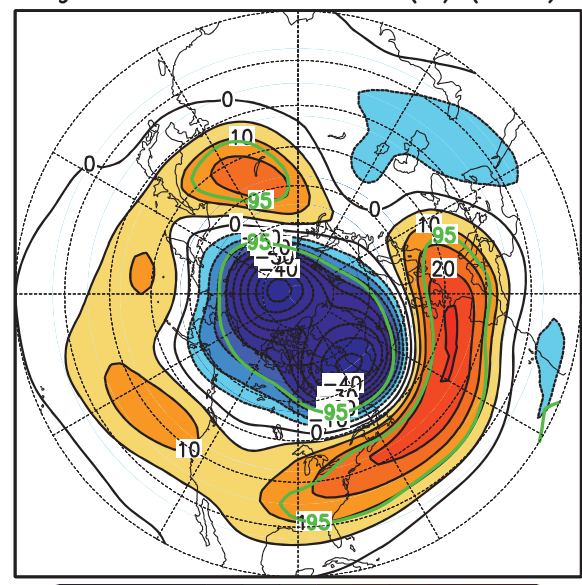

\begin{tabular}{l|c|c|c|c|c|c|cc}
$-40-30-25-20-15-10$ & -5 & 5 & 10 & 15 & 20 & 25 & 30 & 40
\end{tabular}

Regressed 1000hPa T Anoms (SNAM)

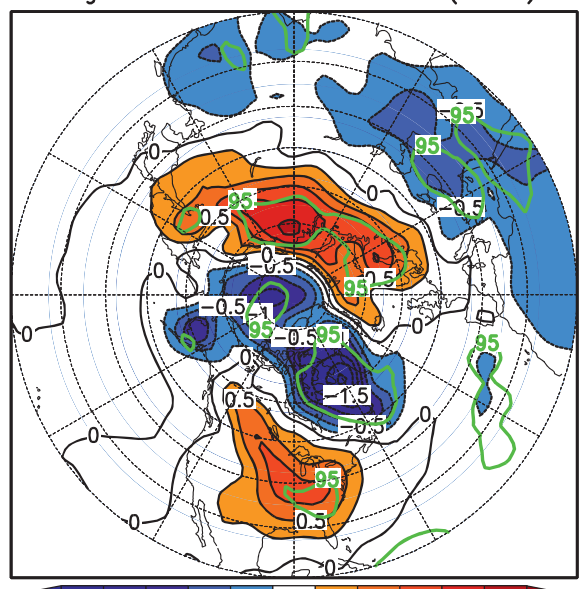

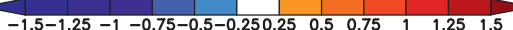

Regressed 50hPa Z Anoms (m) (PAM)

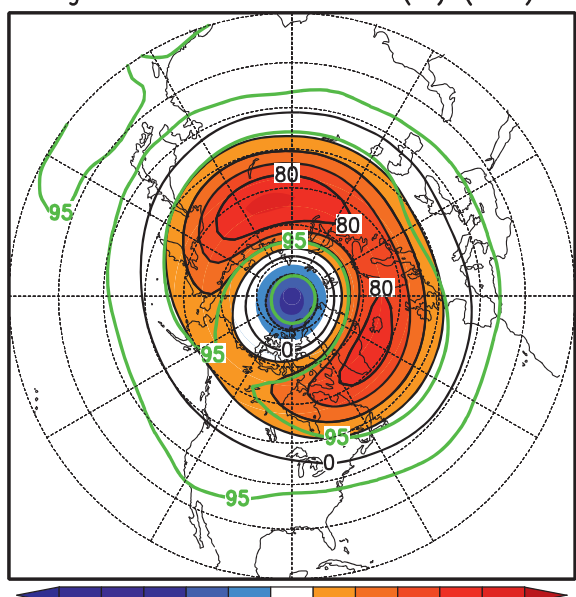

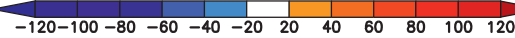

Regressed 500hPa Z Anoms (m) (PAM)

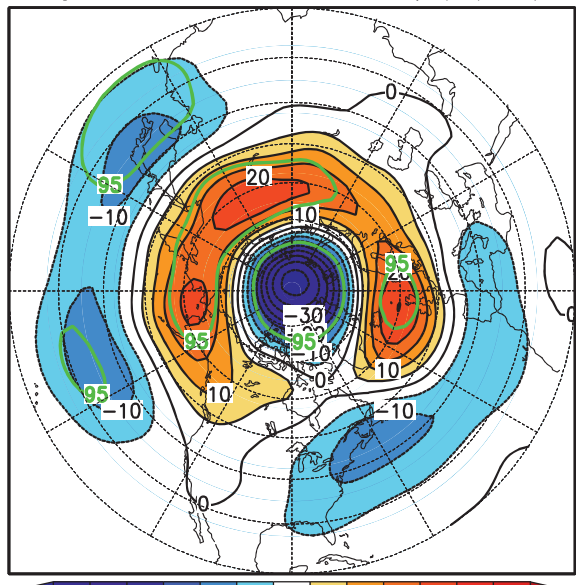

\begin{tabular}{ll|l|l|llllll}
$-40-30-25-20-15-10$ & -5 & 5 & 10 & 15 & 20 & 25 & 30 & 40
\end{tabular}

Regressed 1000hPa T Anoms (PAM)

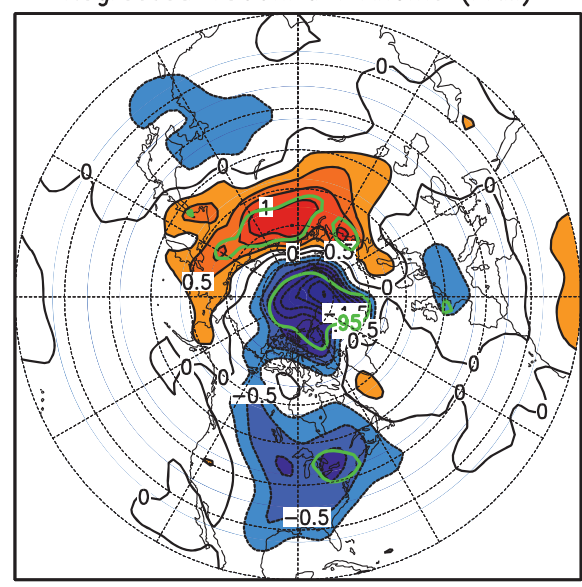

$\begin{array}{lllllll}-1.5-1.25-1 & -0.75-0.5-0.250 .25 & 0.5 & 0.75 & 1 & 1.25 & 1.5\end{array}$

FIG. 4. Linear regressions of boreal winter (JFMA) geopotential height anomalies (m) (top) 50 and (middle) $500 \mathrm{hPa}$, and (bottom) 1000-hPa temperature anomalies (K) against the daily (left) SNAM and (right) PAM indexes. The $95 \%$ confidence level contour is plotted in green. 
canonical pattern. This is consistent with the idea that the tropospheric adjustment to stratospheric NAM events is concentrated over the North Atlantic region.

The regression analysis for the near-surface temperature field (third row in Fig. 4) shows that positive SNAM and PAM events result in polar cooling and northern Siberian warming of similar magnitudes. For PAM, the polar cooling is relatively axisymmetric over the Arctic while for SNAM the cooling is shifted westward with a prominent extension to the west of Greenland. Although relatively weak, the two modes have opposing temperature anomaly signatures over the eastern continental United States. Finally, we note that the SNAM pattern found here is qualitatively consistent with the canonical tropospheric NAM result (Fig. 1 of Thompson and Wallace 1998). The main difference is the relative weakness in magnitude of the temperature anomaly pattern in the current analysis. The analyses presented here reveal that the SNAM and PAM events induce large-scale tropospheric circulation anomaly patterns with (i) distinct spatial patterns and (ii) similar amplitudes. This suggests that, in terms of direct impacts, first-order variations in both the strength and position of the stratospheric polar vortex provide comparable alterations to the tropospheric circulation. However, these impacts have differing spatial patterns and time scales. Further, it is apparent that the net tropospheric response to the stratospheric NAM includes an indirect response that is concentrated over the North Atlantic region.

\section{Sensitivity analyses}

We next examine the robustness of our fundamental results by performing sensitivity tests for the principal component analysis. We first test the dependence upon the spatial domain specification by separately (i) extending the lower-latitude boundary to $20^{\circ} \mathrm{N}$ (from $45^{\circ} \mathrm{N}$ ) and (ii) limiting our analysis to a single vertical level $(50 \mathrm{hPa})$ within the domain of interest (but keeping the lower-latitude boundary fixed at $45^{\circ} \mathrm{N}$ ). The PC analysis is then repeated for the daily zonal-mean zonal wind anomaly field. A third test uses the original spatial domain (Fig. 1c) but applies the PC analysis to the daily zonal-mean geopotential height anomaly field. In all three cases we then repeat the linear regression calculations presented in Figs. 1a,b for the first two EOFs. The resulting zonal-mean zonal wind patterns are presented in Fig. 5 (where SNAM and PAM are still used to indicate the first and second EOFs, respectively). In each of the three test cases the patterns associated with the leading EOFs are remarkably similar in both structure and magnitude to those identified in our baseline case (presented in Fig. 1). The primary difference is that the PAM pattern emerging from the third sensitivity analysis (based upon geopotential height) exhibits somewhat less poleward retraction. We conclude that our zonal-mean PC analyses are not sensitive to the details of either our spatial domain specification or the input field variable considered.

It is of further interest to study whether or not we can reproduce our results from a consideration of zonally varying stratospheric anomaly fields on a single vertical level. To test this we perform an additional sensitivity test in which we perform a PC analysis of the zonally varying height anomaly field at $50 \mathrm{hPa}$ (with latitudinal boundaries as in our baseline case). Because, in this case, we are permitting zonal asymmetry, we expect to obtain prominent modes having a wavelike structure with respect to longitude. These are required in order to fully describe spatial variability associated with the vertical propagation of planetary waves through the lower stratosphere. Indeed, we obtain six statistically distinct EOFs in this analysis [North et al. (1982), according to North's rule; see section 2], and several of the leading modes are characterized by either planetary wavenumber 1 (EOF modes 2 and 3) or 2 (EOF modes 4 and 5) with little projection on the zonal-mean flow. Nonetheless, both EOF1 and EOF6 exhibit strongly zonally symmetric anomaly structures at $50 \mathrm{hPa}$. Linear regression results are presented for these two modes in Fig. 6. Although there are certainly differences in regional detail, the regression results bear a striking resemblance to the parallel baseline analyses of the zonalmean flow presented in Figs. 1 and 4. In particular, the zonal-mean manifestation of EOF6 (Fig. 6: third row, right panel) closely resembles the PAM anomaly structures identified in the four other EOF analyses (Figs. 1b and $5 \mathrm{~d}-\mathrm{f}$ ). Furthermore, the PC time series for EOF6 has a correlation of 0.8 with the baseline PAM PC time series. We conclude that PAM-like anomaly structures emerge from a consideration of zonally varying stratospheric anomaly fields in addition to zonal-mean anomaly fields.

\section{Intraseasonal variability and role in stratospheric warming events}

We next examine the characteristic temporal variability of the SNAM and PAM. To identify the characteristic subseasonal times scales, we first perform a spectral decomposition (via a fast Fourier transform analysis) of the unfiltered PC time series for both SNAM and PAM (Fig. 7). ${ }^{1}$ Although no significant spectral peaks are

\footnotetext{
${ }^{1}$ Although, strictly speaking, such an analysis includes information about SNAM and PAM outside of JFMA, the spectrum is dominated by variability within JFMA.
} 
a
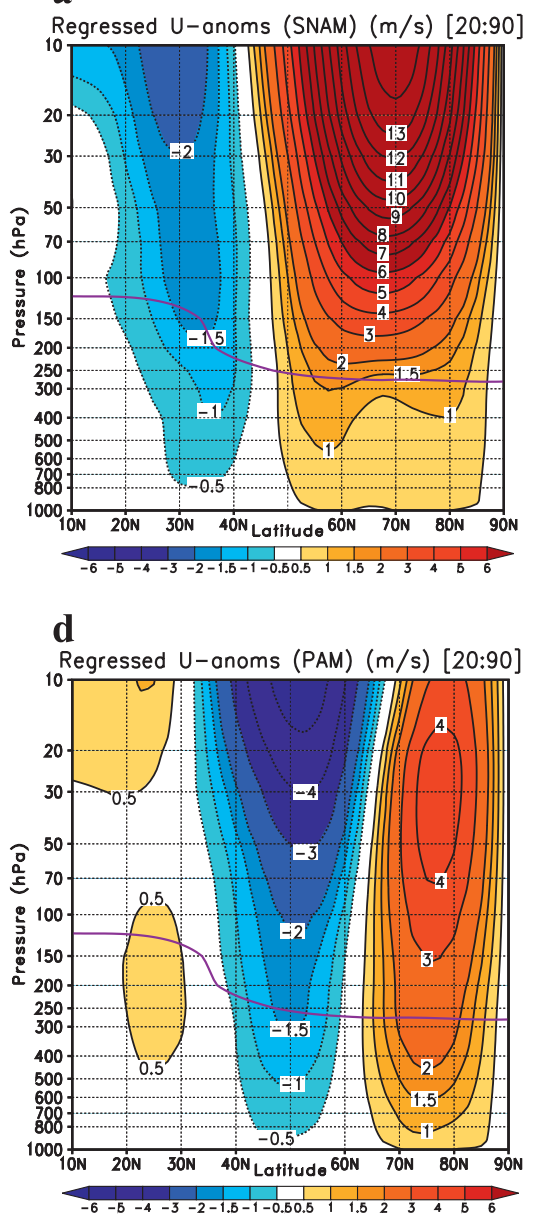

b

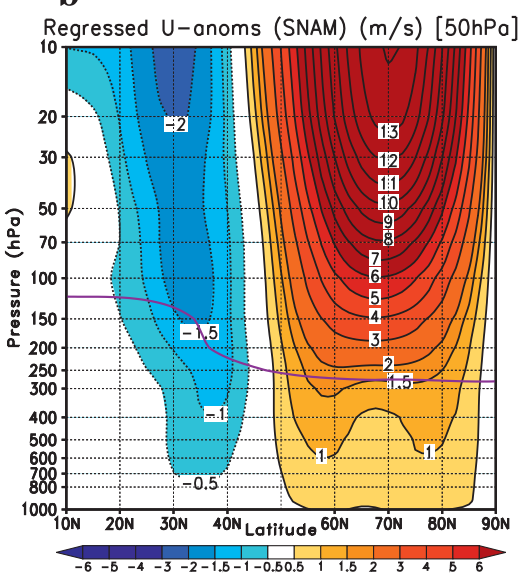

e

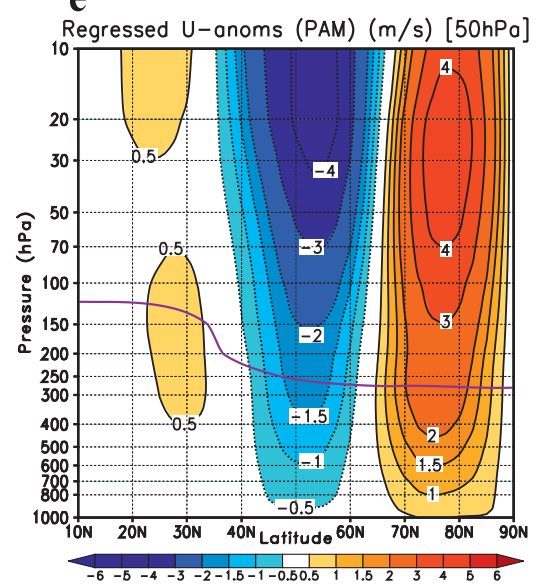

c

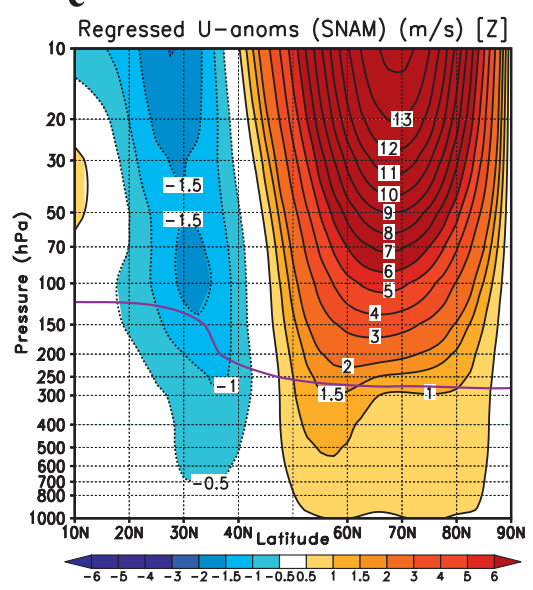

$\mathbf{f}$

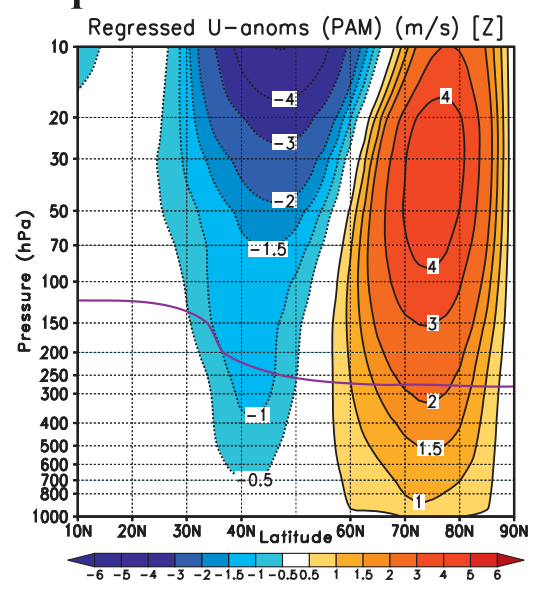

FIG. 5. Sensitivity study displaying the zonally averaged zonal wind field $\left(\mathrm{m} \mathrm{s}^{-1}\right)$. Regressions of boreal winter (JFMA) wind anomalies against the daily SNAM indices calculated using (a) the zonally averaged zonal wind field from $20^{\circ}$ to $90^{\circ} \mathrm{N}$, (b) the zonally averaged wind field at $50 \mathrm{hPa}$, and (c) the zonally averaged height anomaly field. (d)-(f) As in (a)-(c), but for the PAM patterns.

identified, it is nonetheless evident that PAM (SNAM) has more power at shorter (longer) intra-annual time scales. PAM is dominant at periods of less than 50 days, while SNAM dominates at periods of greater than 100 days. The two modes exhibit more equitable power in between these two bounds.

A similar time-scale dichotomy is found that exists in the respective behavior of discrete SNAM and PAM episodes, which is studied via composite analyses of the PC time series. We first study events for which SNAM and PAM occur independently. To do this, we identify times (onset or lag 0 ) when the 5-day running mean of either index first exceeds (falls below) $+1 \sigma(-1 \sigma)$ without a corresponding threshold crossing in the other index within an ensuing 10-day period. We further require events within each of the four classes to be separated by 30 days. This results in 47 (48) positive (negative) SNAM events and 77 (69) positive (negative)
PAM events. Respective lag composite analyses of the PC time series for each event category are shown in Figs. 8a,b. Positive SNAM events are characterized by a relatively gradual development and decay with index values remaining above $+0.5 \sigma$ for a period of $\sim 60$ days. Although negative SNAM events develop more rapidly, they remain below $-0.5 \sigma$ until 30 days after onset. In contrast to SNAM events, PAM events develop and decay relatively quickly, with index magnitudes remaining above $0.5 \sigma$ for less than 2 weeks. Thus, a key result is that the characteristic time scale for discrete PAM events is on the order of 1-2 weeks, which is considerably shorter than that of the SNAM. As such, PAM variability is not only inherently intraseasonal but also includes a substantial submonthly component. Consequently, PAMtype variability is likely to be less evident (or even absent) in similar analyses of monthly mean anomalies [this issue was anticipated by Nigam (1990)]. 
Regressed 50hPa Z Anoms (m) (ZLV1)

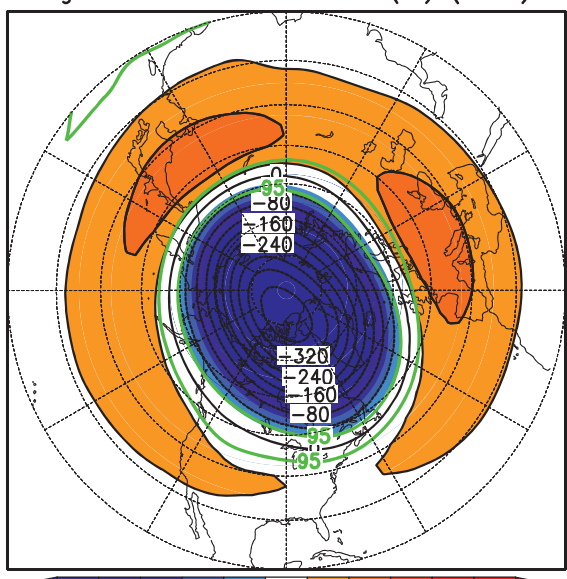

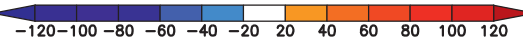

Regressed 500hPa Z Anoms (m) (ZLV1)

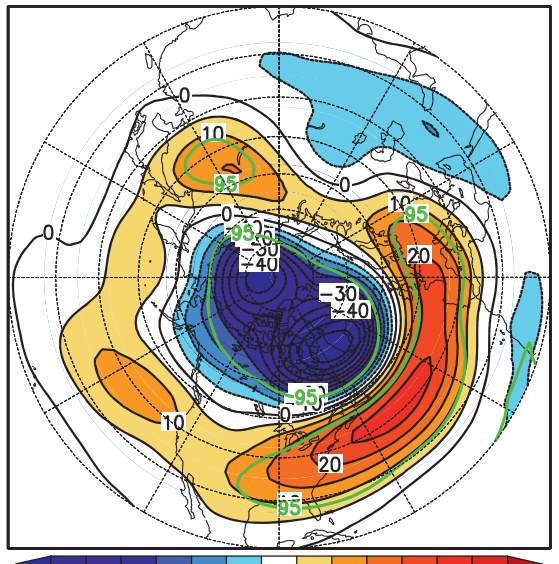

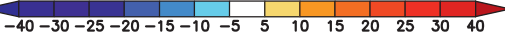

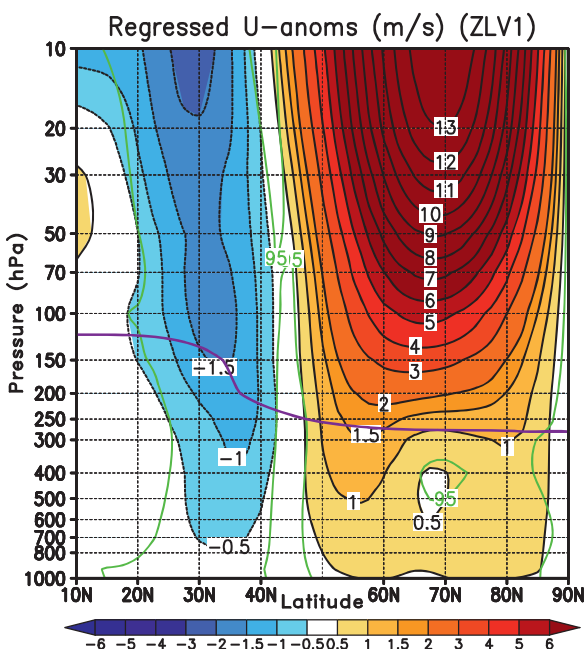

Regressed 50hPa Z Anoms (m) (ZLV6)

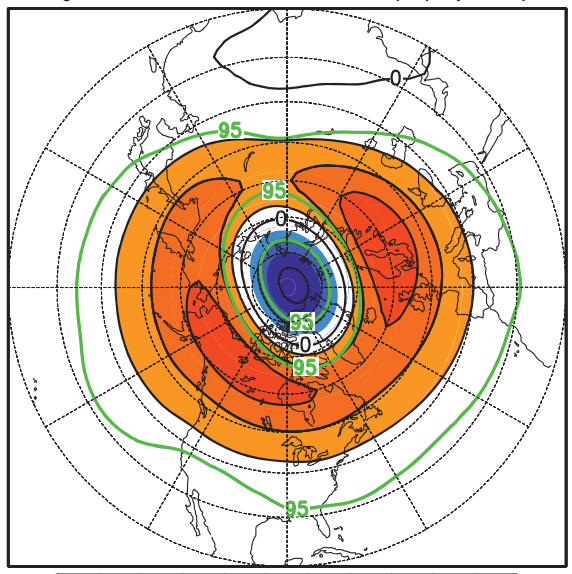

$\begin{array}{lllllllllll}-120-100 & -80 & -60 & -40 & -20 & 20 & 40 & 60 & 80 & 100 & 120\end{array}$

Regressed 500hPa Z Anoms (m) (ZLV6)
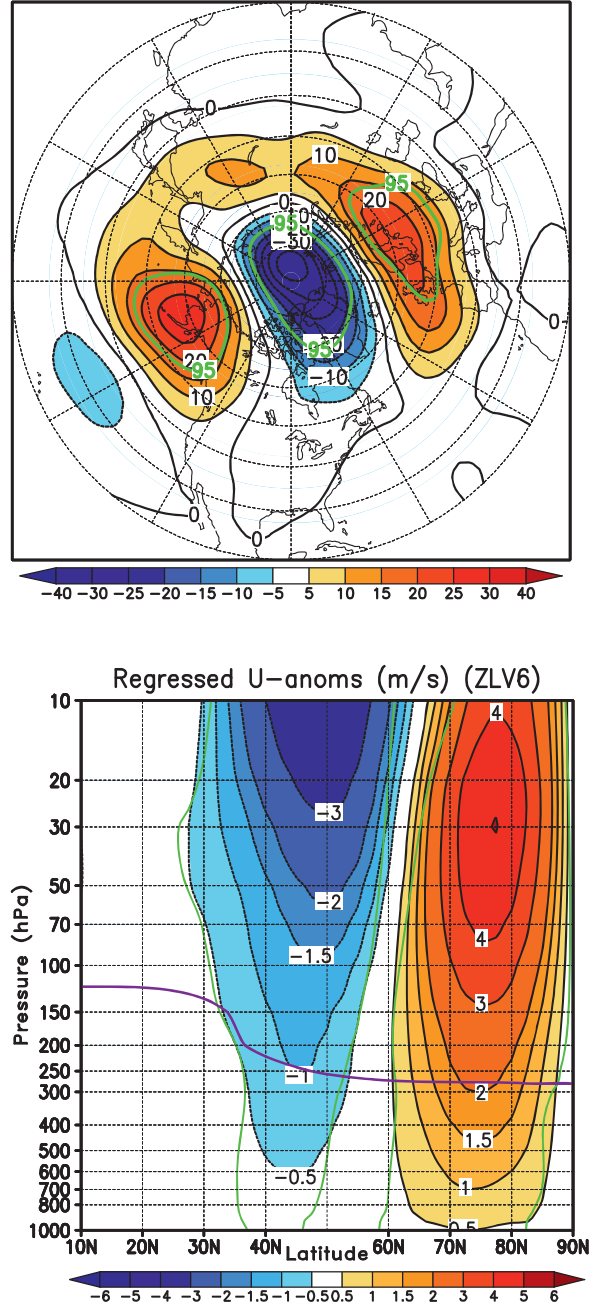

FIG. 6. Linear regressions of boreal winter (JFMA) geopotential height anomalies (m) (top) 50- and (middle) 500-hPa, and (bottom) zonal-mean zonal wind anomalies $\left(\mathrm{m} \mathrm{s}^{-1}\right.$ ) against (left) first PC time series (ZLV1) and (right) sixth PC time series (ZLV6) of the 50-hPa EOF analysis of geopotential height (see text for further details). The $95 \%$ confidence level contour is plotted in green. 
Power Spectrum for SNAM and PAM

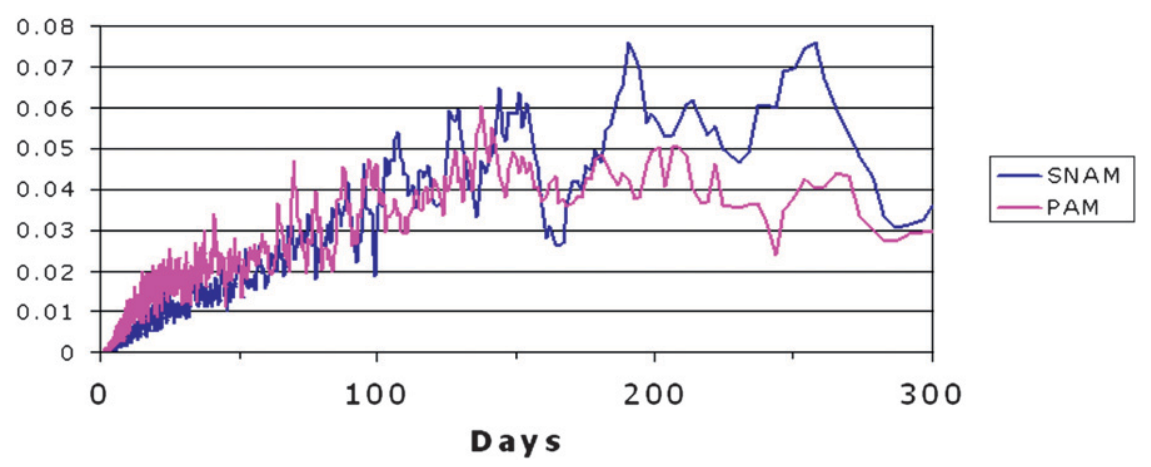

FIG. 7. Characteristic time scale for the boreal wintertime (JFMA) SNAM (blue) and PAM (pink).

We also identify episodes for which like-signed SNAM and PAM events occur at the same time. These are selected to be times when both PC time series first exceed (fall below) $+1 \sigma(-1 \sigma)$. As above, we also require likesigned events to be separated by 30 days, leading to 22 (30) positive (negative) events. The respective lag composite analyses are shown in Figs. 8c,d. Interestingly, the simultaneous occurrence of positive PAM and SNAM events strongly accelerates SNAM decay relative to its occurrence in isolation (Fig. 8a). This juxtaposition of stratospheric zonal circulation anomalies represents a strengthening and poleward shift of the stratospheric polar vortex. This corresponds to the behavior associated with stratospheric preconditioning [which is thought to predispose the stratosphere to SSW events; see Andrews et al. (1987)]. Although less pronounced, similar behavior is observed during simultaneous occurrences of negative SNAM and PAM events (Fig. 8d).

We next investigate the relative roles of SNAM and PAM patterns in the evolution of SSW and SFW events. This is done by simply compositing the SNAM and PAM PC time series with respect to SFW and SSW onset (Figs. $8 \mathrm{e}, \mathrm{f})$. We use the $27 \mathrm{SSW}$ events identified in Charlton and Polvani (2007) and the 47 SFW events considered by BMR. The lag composite analyses reveal that the development and onset of SSW events is dominated by SNAM variability, with the SNAM index dropping below $-1.5 \sigma$ during SSW onset. Both the SNAM and PAM indices exhibit positive tendencies after SSW onset, during which time the polar vortex recovers in strength. We note that PAM appears to lead in this process, indicating that the stratospheric polar vortex recovers more quickly at higher latitudes after SSW events.

In contrast to SSW events, both SNAM and PAM play approximately equal roles in the development, onset, and decay of SFW events. In particular, we note that
SFW events have a precursor evolution that qualitatively mimics the behavior of the simultaneous positive SNAM and PAM events in Fig. 8c. It is evident that the newly identified PAM pattern plays a first-order role in SFW events, which is consistent with the observation by BMR that SFW events have important distinctions from the (stratospheric or tropospheric) NAM. Last, we separate our SSW composite time series analysis into SSW split events (12) and displacement events (15), following the designations of Charlton and Polvani (2007). These results (Figs. 8g,h) indicate that PAM appears to play a larger role in the early stages of splittype SSW events compared to the displacement events. This is consistent with Charlton and Polvani's finding that SSW splitting events are linked to a preconditioning of the stratospheric polar vortex.

\section{Interannual variability and long-term trends}

It is also of scientific interest to study the respective roles of SNAM and PAM in the modulation of the polar vortex on longer time scales. This is motivated partly in response to the Arctic climate paradox discussed in the introduction. There is increasing interest in the potential role of the stratospheric circulation in modulating longterm climate variability (e.g., Son et al. 2008). In the Northern Hemisphere, the polar vortex-climate connection is anticipated to be the strongest during the cool season, when stratosphere-troposphere dynamical coupling is most active (Baldwin et al. 2003). As such, interannual variability in the boreal winter polar vortex has the potential to impact Arctic climate on interannual and longer time scales. In response to the above issues, we perform an exploratory analysis of the long-term variability in the stratospheric polar vortex, which is phrased in terms of the leading EOFs obtained from our 
a

SNAM

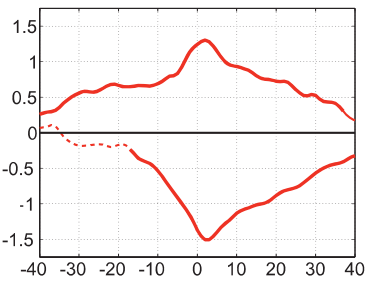

c

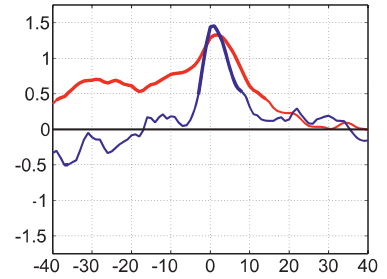

e

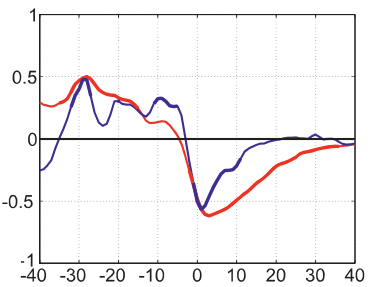

g SSW-Split

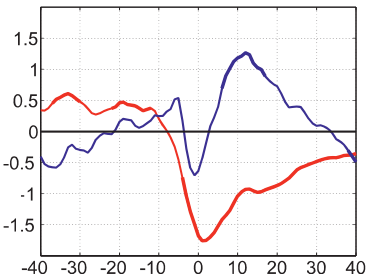

b

PAM

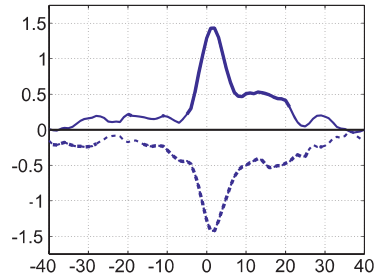

d Both Under

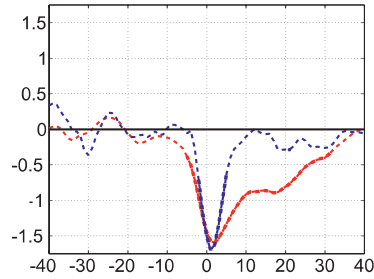

f

SSW

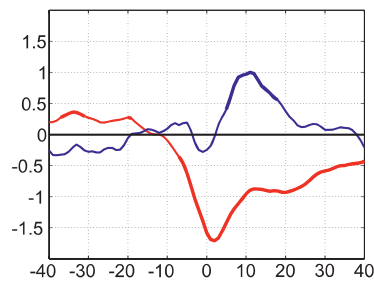

h SSW-Displacement

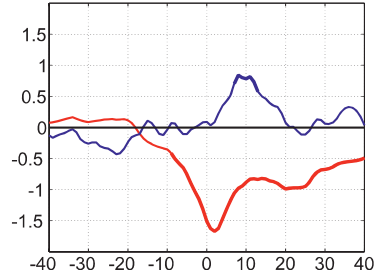

FIG. 8. The composite time evolution of daily unfiltered and normalized SNAM (red) and PAM (blue) indices. Composites of discrete (a) SNAM and (b) PAM events occurring in isolation, and (c),(d) composites for simultaneous, like-signed SNAM and PAM events. (a)-(d) Solid (dashed) lines represent positive (negative) events. Composites with respect to the onset of (e) SFW and (f) SSW events. Composites of SSW events stratified by type: (g) split and (h) displacement. In all cases lag 0 represents the time of event onset (see text for further details). Significant segments are plotted more thickly.

PC analysis. Figure 9 shows the respective interannual anomalies in the SNAM and PAM PC time series (where each data point represents the JFMA average anomaly for the calendar year). For comparison, the SNAM PC time series is directly contrasted with the analogous time series for the canonical 50-hPa NAM (Baldwin and Dunkerton 2001). There are the following several points to note regarding the year-to-year variability in SNAM/NAM:

1) SNAM and the canonical 50-hPa NAM track each other very well.

2) Both series reveal a long-term NAM trend between $\sim 1970$ and $\sim 1995$ (as discovered by Thompson et al. 2000).

3) There is a dramatic reversal in this trend in more recent years.

4) Over the 49-yr time period considered, neither SNAM nor the canonical 50-hPa NAM has a statistically significant trend.

In contrast to SNAM, the year-to-year variability in the PAM index includes a statistically significant [at the 99\% confidence level, following Aiken and West (1991)] trend in which PAM has shifted from a positive phase toward a more predominantly negative phase (Fig. 9b). This trend is consistent with a long-term southward shift in (and high latitude weakening of) the polar vortex (recall Fig. 2b) over the past $50 \mathrm{yr}$. Although some of this long-term variability may be linked to a stratospheric regime "shift" in the late 1970s (e.g., Christiansen 2003), the linear trend accounts for $66 \%$ of the year-to-year variability. To further explore the role of SNAM and PAM structures in the long-term trend of the stratospheric polar vortex, we next decompose existing trends in the zonal-mean zonal wind into parts that are "linearly congruent" with either SNAM or PAM, respectively (following the methods of Thompson et al. 2000). The linear trend and interannual standard deviation in the zonal-mean zonal wind are displayed in the top panels of Fig. 10. Although the trend pattern at subtropical latitudes is rather noisy (and associated with a large standard deviation), at higher latitudes within the stratosphere there is a coherent north-south dipole pattern of zonal accelerations (decelerations) at middle (high) latitudes. Again, this zonal wind anomaly pattern is consistent with a southward shift in the stratospheric polar vortex. We then use the results of our PC analysis in order to determine the respective contributions of SNAM and PAM to the long-term trend pattern (e.g., Thompson et al. 2000). These results are displayed in the bottom frames of Fig. 10. Although SNAM appears to provide a weak long-term strengthening of the polar vortex, the long-term trend pattern has more strong associations with the negative phase of the PAM pattern. We conclude that PAM plays a major role in describing the long-term trend in the boreal stratospheric polar vortex, which is characterized by a modest southward shift in the polar vortex location. Thus, PAM (and/or the latitudinal position of the boreal polar vortex) may be an 
Long-Term Stratospheric NAM Trend

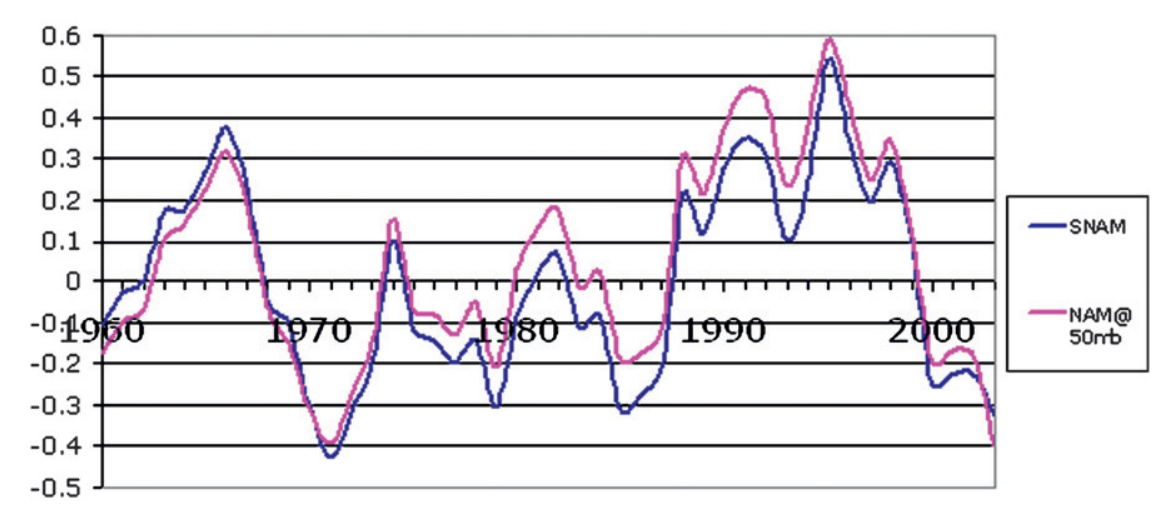

Long-Term PAM Trend

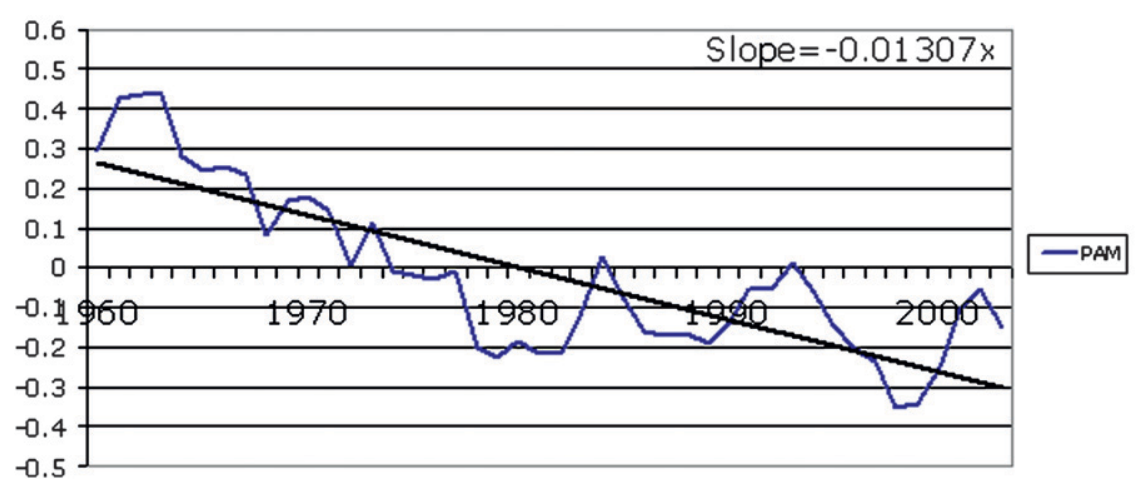

FIG. 9. Long-term trends in the wintertime-averaged (JFMA) NAM and PAM. (top) The SNAM (blue) and the NAM (pink) index at $50 \mathrm{mb}$. (bottom) The long-term trend in the PAM. All data plotted have been lightly smoothed using a five-point running mean.

important stratospheric signature to look for in climate change "fingerprinting" (because most studies focus on long-term variability in the behavior of NAM).

\section{Conclusions}

Intraseasonal variability in the boreal stratospheric polar vortex is characterized using a principal component (PC) analysis of daily anomalies in the zonal-mean zonal wind field within the high-latitude lower stratosphere. The leading modes are associated with vertically coherent north-south dipoles in the zonal wind field extending from the midstratosphere down to the earth's surface. The first mode, referred to as SNAM, represents intraseasonal variability in polar vortex strength and is highly correlated with the canonical stratospheric NAM. The second mode, referred to as the polar annular mode (PAM), represents intraseasonal variability in the latitudinal position of the polar vortex and is structurally and statistically distinct from SNAM. In its positive (negative) phase PAM is associated with a northward (southward) shift in the polar vortex position with below (above)-normal heights over the pole. PAM also provides important alterations to the zonal-mean zonal wind structure along the tropopause at middle to high latitudes. Sensitivity analyses indicate that the main results of our PC analysis are robust to the (i) spatial domain specification, (ii) input field variable considered, and (iii) use of zonal-mean or zonally varying anomaly fields.

Linear regression analyses show that, at both stratospheric and tropospheric altitudes, the PAM anomaly pattern is retracted substantially northward in comparison to the SNAM. Nonetheless, PAM events are associated with circumpolar circulation anomaly patterns in the high-latitude troposphere with magnitudes that are comparable to (and even exceed) SNAM. In addition, the positive phase of PAM leads to 

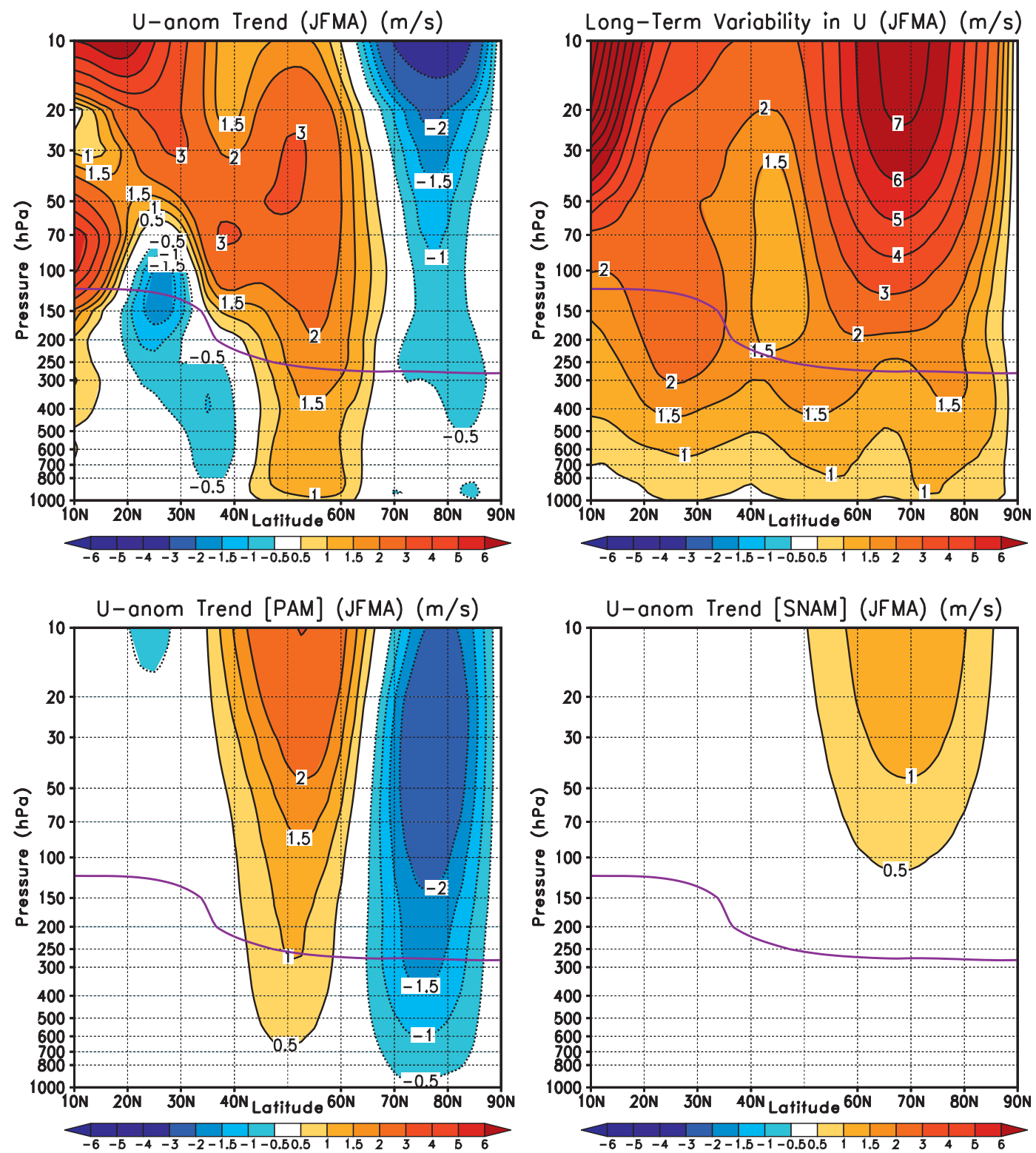

FIG. 10. Long-term trends in the zonally averaged zonal wind field $\mathrm{m} \mathrm{s}^{-1}$ over 48 years (top) The long-term trend in the (left) JFMA-averaged zonal wind field as well as the (right) long-term variability represented by the standard deviation. (bottom) The contribution to the trend resulting from the trends in (left) PAM and (right) SNAM.

colder (warmer) surface air temperatures over the Arctic (northern Siberia). Composite analyses of the respective $\mathrm{PC}$ time series indicate that PAM events are relatively short lived (1-2 weeks) compared to SNAM. However, when SNAM and PAM events of the same sign occur simultaneously, they synergistically interact in such a way as to hasten the demise of the SNAM. For positive phases, the concomitant strengthening and tightening of the polar vortex may predispose the vortex to an abrupt decay via the stratospheric preconditioning process (Andrews et al. 1987). We also find that the evolution of sudden stratospheric warming events is dominated by SNAM variability, whereas SNAM and PAM play approximately equal roles in stratospheric final warming.

An important new result of our study is the finding that stratospheric and tropospheric NAM variability is largely decoupled on daily time scales, with SNAM accounting for less than $25 \%$ of the daily tropospheric NAM variability. Consistent with this result, we also find that the direct tropospheric manifestation of SNAM (as determined via linear regression analysis) has only a 
modest correspondence to the canonical tropospheric NAM pattern (Thompson and Wallace 2000). The tropospheric anomaly patterns obtained in our study are more strongly annular with relatively weak amplitudes over the North Atlantic sector. Taken together, the two above results are consistent with the idea that the proximate tropospheric manifestation of SNAM differs from the canonical tropospheric NAM pattern. This suggests that, during periods of stratosphere-troposphere NAM coupling, the ultimate net tropospheric response has a substantial component representing an internal tropospheric adjustment to the SNAM variability [e.g., the indirect tropospheric response discussed by McDaniel and Black (2005)]. As such, the current analysis may provide a useful dynamical model for delineating stratospheric and tropospheric NAM variability and their coupling. This separation also serves to isolate the direct impact of the polar vortex variability upon the troposphere. Because SNAM and PAM induce large-scale tropospheric circulation anomaly patterns with similar amplitudes, our results suggest that firstorder variations in the strength and position of the stratospheric polar vortex provide alterations to the tropospheric circulation of comparable strength. However, the respective tropospheric impacts have different spatial patterns and time scales. Finally, our results also reveal that recent decadal trends in the boreal stratospheric polar vortex project more strongly onto PAM than SNAM.

In summary, we conclude that the polar annular mode represents a newly recognized annular mode that (i) strongly couples the troposphere and stratosphere on submonthly time scales, (ii) provides a robust circumpolar circulation in the high-latitude troposphere, and (iii) plays an essential role in stratospheric final warming events as well as long-term trends in the boreal stratospheric polar vortex. Future research efforts will use the current paradigm as a framework for exploring stratosphere-troposphere coupling on submonthly time scales.

Acknowledgments. This research was supported by the NSF Climate and Large-Scale Dynamics Program under Grant ATM-0456157 (under the U.S. CLIVAR Program). The NCEP-NCAR reanalyses come from the NOAA/Climate Diagnostics Center from their Web site (online at http://www.cdc.noaa.gov/). We thank Dr. Steven Feldstein and two anonymous reviewers for their helpful comments and guidance on our paper.

\section{REFERENCES}

Aiken, L., and S. West, 1991: Multiple Regression: Testing and Interpreting Interactions. Sage Publications, $224 \mathrm{pp}$.
Andrews, D. G., J. R. Holton, and C. B. Leovy, 1987: Middle Atmosphere Dynamics. Academic Press, 489 pp.

Baldwin, M. P., and T. J. Dunkerton, 1999: Propagation of the Arctic Oscillation from the stratosphere to the troposphere. J. Geophys. Res., 104, 30 937-30946.

—, and -2001 : Stratospheric harbingers of anomalous weather regimes. Science, 294, 581-584.

—, D. B. Stephenson, D. W. J. Thompson, T. J. Dunkerton, A. J. Charlton, and A. O'Neill, 2003: Stratospheric memory and skill of extended-range weather forecasts. Science, 301, 636-640.

Black, R. X., and B. A. McDaniel, 2007: The dynamics of Northern Hemisphere stratospheric final warming events. J. Atmos. Sci., 64, 2932-2946.

,-- , and W. A. Robinson, 2006: Stratosphere-troposphere coupling during spring onset. J. Climate, 19, 4891-4901.

Bromwich, D. H., and S.-H. Wang, 2008: A review of the temporal and spatial variability of Arctic and Antarctic atmospheric circulation based upon ERA-40. Dyn. Atmos. Oceans, 44, 213-243.

Charlton, A. J., and L. M. Polvani, 2007: A new look at stratospheric sudden warmings. Part I: Climatology and modeling benchmarks. J. Climate, 20, 449-469.

Christiansen, B., 2003: Evidence for nonlinear climate change: Two stratospheric regimes and a regime shift. J. Climate, 16, 3681-3689.

Itoh, H., and K. Harada, 2004: Coupling between tropospheric and stratospheric leading modes. J. Climate, 17, 320-336.

Kalnay, E., and Coauthors, 1996: The NCEP/NCAR 40-Year Reanalysis Project. Bull. Amer. Meteor. Soc., 77, 437-471.

Limpasuvan, V., D. W. J. Thompson, and D. L. Hartmann, 2004: The life cycle of Northern Hemisphere stratospheric sudden warmings. J. Climate, 17, 2584-2596.

Lorenz, D. J., and D. L. Hartmann, 2003: Eddy-zonal flow feedback in the Northern Hemisphere winter. J. Climate, 16, 1212-1227.

Maslanik, J., S. Drobot, C. Fowler, W. Emery, and R. Barry, 2007: On the Arctic climate paradox and the continuing role of atmospheric circulation in affecting sea ice conditions. Geophys. Res. Lett., 34, L03711, doi:10.1029/2006GL028269.

McDaniel, B. A., and R. X. Black, 2005: Intraseasonal dynamical evolution of the northern annular mode. J. Climate, 18, 3820-3839.

Nigam, S., 1990: On the structure of variability of the observed tropospheric and stratospheric zonal-mean zonal wind. J. Atmos. Sci., 47, 1799-1813.

North, G. R., T. L. Bell, R. F. Cahalan, and F. J. Moeng, 1982: Sampling errors in the estimation of empirical orthogonal functions. Mon. Wea. Rev., 110, 699-706.

Overland, J. E., and M. Wang, 2005: The Arctic climate paradox: The recent decrease of the Arctic Oscillation. Geophys. Res. Lett., 32, L06701, doi:10.1029/2004GL021752.

Robinson, D. P., R. X. Black, and B. A. McDaniel, 2006: A Siberian precursor to midwinter intraseasonal variability in the North Pacific storm track. Geophys. Res. Lett., 33, L15811, doi:10.1029/ 2006 GL026458.

Son, S. W., and Coauthors, 2008: The impact of stratospheric ozone recovery on the Southern Hemisphere westerly jet. Science, 320, $1486-1489$.

Song, J., W. Zhou, J. Pan, and C. Li, 2006: Global influence of the Northern Hemisphere second mode of the zonal average of the zonal wind. Geophys. Res. Lett., 33, L18703, doi:10.1029/ 2006GL026380. 
Thompson, D. W. J., and J. M. Wallace, 1998: The Arctic Oscillation signature in the wintertime geopotential height and temperature fields. Geophys. Res. Lett., 25, 1297-1300.

— and - 2000: Annular modes in the extratropical circulation Part I: Month-to-month variability. J. Climate, 13, 1000-1016.

—, and —, 2001: Regional climate impacts of the Northern Hemisphere annular mode. Science, 293, 85-89.

,-- , and G. C. Hegerl, 2000: Annular modes in the extratropical circulation. Part II: Trends. J. Climate, 13, 1018-1036.
Wilks, D. S., 2006: Statistical Methods in the Atmospheric Sciences. Academic Press, 627 pp.

Wittman, M. A. H., A. J. Charlton, and L. M. Polvani, 2005: On the meridional structure of annular modes. J. Climate, 18, 21192122.

Wu, Q., and D. J. Karoly, 2007: Implications of changes in the atmospheric circulation on the detection of regional surface air temperature trends. Geophys. Res. Lett., 34, L08703, doi:10.1029/2006GL028502. 